Research Article

\title{
Transient Behavior of a Prototype Centrifugal Pump with Abrupt Decreasing Flow Rate
}

\author{
Yan-Juan Zhao $\mathbb{D}^{1}$ and Yu-Liang Zhang $\mathbb{D}^{2}$ \\ ${ }^{1}$ College of Information Engineering, Quzhou College of Technology, Quzhou 324000, China \\ ${ }^{2}$ College of Mechanical Engineering, Quzhou University, Quzhou 324000, China \\ Correspondence should be addressed to Yu-Liang Zhang; zhang002@sina.com
}

Received 24 February 2021; Revised 3 April 2021; Accepted 19 April 2021; Published 24 April 2021

Academic Editor: Evgeny Petrov

Copyright (c) 2021 Yan-Juan Zhao and Yu-Liang Zhang. This is an open access article distributed under the Creative Commons Attribution License, which permits unrestricted use, distribution, and reproduction in any medium, provided the original work is properly cited.

\begin{abstract}
Centrifugal pump often operates at different working flow rates to meet engineering requirement. To better reveal the transient behavior of centrifugal pump in the process of decreasing flow rate, the finite volume method (FVM), RNG $k-\varepsilon$ turbulence model, sliding mesh technology, and user-defined functions (UDF) were employed to simulate the three-dimensional unsteady viscous incompressible flow in a low-specific-speed centrifugal pump during the abrupt valve-off period. The results show that the differences are very obvious between transient and quasi-steady calculations. The velocity is maximum on the wall of hub and shroud, while the velocity is minimum and uniform distribution at middle positions. The transient flow field lags behind the quasisteady flow field, which may be related to the reasons; namely, kinetic energy cannot convert pressure energy in time.
\end{abstract}

\section{Introduction}

Centrifugal pump is widely used, and many scholars at home and abroad have deeply researched and obtained plentiful achievements. This research all belongs to stable states; namely, rotational speed or piping system load is invariable or has a slight change. So far, most of the research mainly concentrates on the above region. Except for stable states, many unstable states are also existent, such as rapid startup, stopping accident, rapid regulating flow rate, and so forth. With the development of the application field, for example, underwater weapon launch, rapid startup of the large pump station, it is more and more necessary to research transient behavior.

Tsukamoto and Ohashi studied the transient characteristics of a centrifugal pump during a rapid starting and stopping period by theoretical analysis and experimental study $[1,2]$. They think that the impulsive pressure and the lag in circulation formation around impeller vanes were the main reasons for the difference between dynamic and quasi-steady characteristics. Tsukamoto et al. studied the dynamic characteristics of centrifugal pump subject to sinusoidal changes in rotational speed [3]. The model actually is similar to rotational speed control by frequency variation. It was found that, with the increased frequency of rotational speed fluctuations, the difference between dynamic and quasi-steady characteristics was more remarkable. Lefebvre and Barker tested four transient cases of a mixed-flow pump during the acceleration and deceleration period [4]. Results show the quasi-steady hypothesis in predicting the pump performance is unreliable. Thanapandi and Prasad tested a volute pump with different valve openings to study the dynamic behavior of the pump during normal startup and stopping [5]. The results showed that the characteristic during the common transient period was closed to quasi-steady. They also originally analyzed the dynamic performances using the method of characteristics. Antoine Dazin et al. presented a method of predicting the turbomachinery's transient behavior [6]. Based on the angular momentum and energy equations, the internal torque, power, and head could be solved. Results showed that the behavior of a pump impeller is not only depending on the acceleration rate and flow rate, as it is usually admitted, but also on velocity profiles and their evolution during the transient. Tanaka and Tsukamoto tested the transient behavior of a cavitating centrifugal pump at the sudden opening or closure of the discharge valve and quick starting or stopping of the pump [7-9]. 
They found that unsteady pressure, as well as flow rate, is related to the time-dependent cavitation behavior. Pressure and flow rate fluctuations were found to occur due to oscillating cavitation or water column separation at rapid transient operations. Experimental results indicated that transient phenomena at the opening of discharge valve and pump starting were quite different from those at the closing of discharge valve and pump stopping. They introduced a new unsteady parameter to judge the occurrence of the fluctuating pressure and flow rate during the transient process. Li et al. adopted a new dynamic slip region method to simulate the three-dimensional unsteady incompressible viscous flows in a centrifugal pump during a rapid starting period [10]. The pump was included in a pipe system, so boundary conditions could be omitted. Wang et al. studied the transient behavior of centrifugal pump and mixed-flow pump during rapid starting by numerical simulation and experiment [11-15]. They found the transient behavior is very remarkable. Wu et al. studied the external transient hydrodynamic performance and the internal flow mechanism of the centrifugal pump by numerical simulation and experiment during the discharge valve rapid opening [16]. What is more, other some important studies also showed different unsteady flow characteristics in pump or turbine [17-23]. Zhang et al. studied transient behavior in prototype centrifugal pump during rapid regulating flow rate using CFD [24]. Based on the previous study, the flow characteristics need to be more deeply researched so as to better reveal transient characteristics.

In the above research, transient behaviors are very obvious. By far, research about transient behaviors is very limited. In this paper, the transient behavior in a low-specific-speed centrifugal pump will be researched during the rapid shutting down valve period by numerical simulation. In the process of shutting down valve, the rotational speed is nearly constant, while the flow rate is a fast variable.

\section{Physical Model}

In the piping system with centrifugal pump, when the flow rate is increased by regulating valve, pump head possibly reaches zero; this is an utmost working point. When the flow rate is decreased, the pump head possibly reaches the maximum; this is also an utmost point. In order to analyze the transient behavior during regulating flow rate, a lowspecific-speed centrifugal pump is used as a calculation object. The main design parameters are as follows: the flow rate is $6 \mathrm{~m}^{3} / \mathrm{h}$, the head is $8 \mathrm{~m}$, the rotational speed is $1450 \mathrm{r} /$ min. The suction and discharge diameters of the pump are $50 \mathrm{~mm}$ and $40 \mathrm{~mm}$. The inlet and outlet diameters of the impeller are $48 \mathrm{~mm}$ and $160 \mathrm{~mm}$. The inlet and outlet widths of the impeller are $20 \mathrm{~mm}$ and $10 \mathrm{~mm}$. The blade angles at inlet and outlet are $25^{\circ}$, and the blade number is 5 . The grid in the calculation region could be seen in Figure 1.

\section{Numerical Method}

3.1. Control Equations. The continuity equation and Navier-Stokes equations of the three-dimensional unsteady viscous flow in the centrifugal pump are as follows:

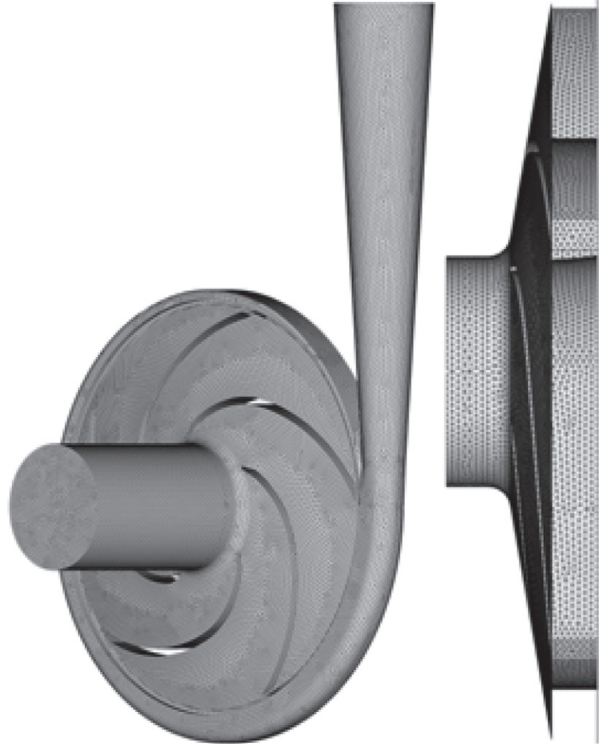

Figure 1: Grid of calculation region.

$$
\begin{gathered}
\frac{\partial \rho}{\partial t}+\frac{\partial\left(\rho u_{i}\right)}{\partial x_{i}}=0 \\
\frac{\partial\left(\rho u_{i}\right)}{\partial t}+\frac{\partial\left(\rho u_{i} u_{j}\right)}{\partial x_{j}}=\frac{\partial}{\partial x_{j}}\left(\mu \frac{\partial u_{i}}{\partial x_{j}}-\rho \overline{u_{i}^{\prime} u_{j}^{\prime}}\right)-\frac{\partial p}{\partial x_{i}}+S_{i} .
\end{gathered}
$$

In order to seal the equations and aim at the Reynolds stress in the time-mean equation, the turbulence model has to be adopted to establish a link between the time-mean valve and pulse value. $k-\varepsilon$ two-equation model is widely applied. RNG $k-\varepsilon$ turbulence model in the eddy viscosity models will be adopted in this paper, which has made sure that it is well suited to the interior flow of the pump and welldisposed flow of high strain rate and high curve degree [25]. The constraint equations of $k$ and $\varepsilon$ are as follows:

$$
\begin{aligned}
& \frac{\partial(\rho k)}{\partial t}+\frac{\partial\left(\rho k u_{i}\right)}{\partial x_{i}}=\frac{\partial}{\partial x_{j}}\left(\alpha_{k} \mu_{\mathrm{eff}} \frac{\partial k}{\partial x_{j}}\right)+G_{k}+\rho \varepsilon, \\
& \frac{\partial(\rho \varepsilon)}{\partial t}+\frac{\partial\left(\rho \varepsilon u_{i}\right)}{\partial x_{i}}=\frac{\partial}{\partial x_{j}}\left(\alpha_{\varepsilon} \mu_{\mathrm{eff}} \frac{\partial \varepsilon}{\partial x_{j}}\right)+\frac{C_{1 \varepsilon}^{*} \varepsilon}{k} G_{k}-C_{2 \varepsilon} \rho \frac{\varepsilon^{2}}{k},
\end{aligned}
$$

where $\mu_{\text {eff }}=\mu+\mu_{t}, \mu_{t}=\rho C_{\mu}\left(k^{2} / \varepsilon\right), C_{1 \varepsilon}^{*}=C_{1 \varepsilon}-(\eta(1-(\eta / \eta$ $\left.\left.\left.{ }_{0}\right)\right) / 1+\beta \eta^{3}\right), \eta=\left(2 E_{i j} \cdot E_{i j}\right)^{1 / 2}(k / \varepsilon)$, and $E_{i j}=(1 / 2)\left(\left(\partial u_{i} / \partial\right.\right.$ $\left.\left.x_{j}\right)+\left(\partial u_{j} / \partial x_{i}\right)\right)$.

The constant values in the model are as follows: $C_{\mu}=0.0845, \alpha_{k}=\alpha_{\varepsilon}=1.39, C_{1 \varepsilon}=1.42, C_{2 \varepsilon}=1.68, \quad \eta_{0}=$ 4.377, and $\beta=0.012$.

3.2. Sliding Mesh. In order to obtain interior flow characteristics, the sliding mesh technology is used, and its principle is shown in Figure 2. When the calculation domain is meshed, it is divided into several subdomains. Sliding mesh technology makes bilateral meshes slide on the interface, and does not require mesh points superposition. But 


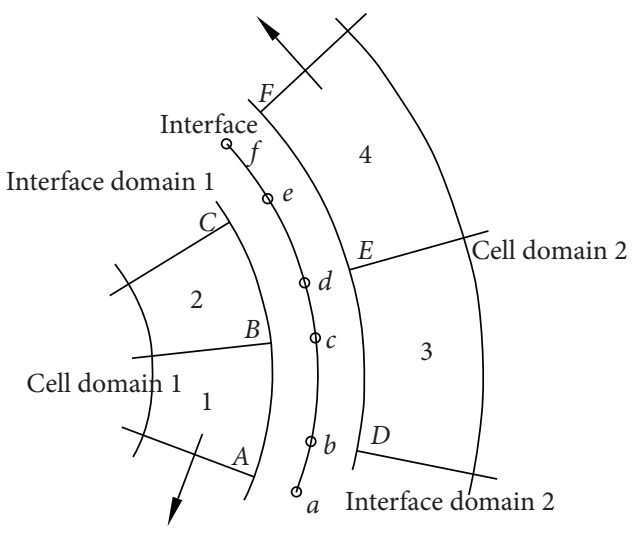

Figure 2: Sliding mesh principle.

bilateral flux must be calculated and make them equal. When sliding mesh passes datum through an interface, all meshes are not rebuilt. The calculation model is common control equations. After iteration in every time step, the whole rotation domain begins to slide in the light of the appointed mode and then continues to calculate in the next time step.

3.3. Calculation Method. In order to precisely obtain interior flow characteristics during regulating flow rate, finite volume method, sliding mesh technology, and user-defined functions (UDF) were applied to simulate the three-dimensional unsteady viscous flow. At inlet, the boundary condition and initial condition are simultaneously given by UDF. The flow rate is regulated from the design flow rate to zero; namely, the valve is shut down, and the flow rate is uniform change. The regulating time is $0.1 \mathrm{~s}$ :

$$
Q(t)= \begin{cases}6, & t<t_{0} \\ 6-60\left(t-t_{0}\right), & t_{0} \leq t \leq t_{0}+0.1 \\ 0, & t>t_{0}+0.1\end{cases}
$$

where $Q(t)$ is the transient flow rate $\left(\mathrm{m}^{3} / \mathrm{h}\right) . t_{0}$ is stable operation time before regulating flow rate.

At outlet, the outflow condition is applied. The assumption is that the flow is completely developed at any moment; namely, every parameter does not change along the flow direction:

$$
\begin{cases}\frac{\left.\partial u_{j}\right|_{\text {out }}}{\partial \vec{n}}=0, & \frac{\partial p_{\text {out }}}{\partial \vec{n}}=0, \\ \frac{\partial k_{\text {out }}}{\partial \vec{n}}=0, & \frac{\partial \varepsilon_{\text {out }}}{\partial \vec{n}}=0, \\ & j=1,2,3 .\end{cases}
$$

No slip boundary condition is adopted on the wall. Standard wall function is also adopted to dispose the low Reynolds number problem near the wall region. The oneorder upwind form is adopted to discrete convection term, the central difference form with two-order accuracy is adopted to discrete diffusion term, the standard form is also adopted to discrete source term. The SIMPLE algorithm is used to couple velocity and pressure. Flow medium is water, density is $1000 \mathrm{~kg} / \mathrm{m}^{3}$, dynamic viscosity is $1.0 \times 10^{-3} \mathrm{~Pa} \cdot \mathrm{s}$, and gravity is taken into account. The calculation domain is meshed by an unstructured grid. The dependence test of the grid number is also carried out with different grid numbers. It is found that when the relative error of the predicted head is less than $2 \%$, the grid number effect can be ignored. Although the predicted head shows slight rising tendency with the increase of grid numbers, the evolution tendency of the flow field remains the same. Meanwhile, the final grid number is 508792 so as to keep high calculating efficiency. The present grid number is insufficient to simulate the microcosmic flow like in the boundary layer, but it is able to be used to correctly predict the external performance and capture the basic flow phenomenon. Subsequently, the grid quality is checked and the results show that "EquiAngle Skew" and "EquiSize Skew" of all grids are less than 0.85 and the corresponding maximum values of $y+$ on wall surfaces are within the range of 35 ; therefore, the grid quality is also satisfied.

The effect of time step on unsteady numerical results is studied to validate the independence of time step. Three time steps, that is, $0.0001379 \mathrm{~s}, 0.0002069 \mathrm{~s}$, and $0.0004138 \mathrm{~s}$, are used in the simulation. These time steps correspond to 300 , 200 , and 100 time steps in a revolution, respectively. The numerical simulation results show that the relative error of the predicted head is less than $2 \%$; therefore, the computational time step size was selected as $0.0004138 \mathrm{~s}$ so as to save the calculating time. The average Courant number in the transient simulations is about 3. In software ANSYSFLUENT, the default iteration number in a time step is 20 . Actually, this iteration number is insufficient for most of the calculating projects. In order to ensure convergence, the set iteration number in a time step should be as more as possible. In order to save the total calculating time, the set iteration number should be as less as possible. Therefore, there are the minimum and maximum loops in each time step in theory. In the present work, the iteration numbers for each time step were set to 2000 (the maximum number of iterations for each time step actually only are about 40 ) so as to make ensure of the convergence. During the iterations process, the convergence history curves for continuity and momentum equations for each time step were below 0.0001 . The required time to calculate the whole flow is about 45 hours by means of 10 processors in a computer. The reliability of numerical results has been validated in [26].

\section{Result Analysis}

4.1. Stable Operating State. The velocity distributions at different sections ( $r=72 \mathrm{~mm})$ are shown in Figure 3, wherein the symbol $s$ stands for the distance from the hub to any point along the axial direction in the impeller and $b$ indicates the distance from the hub to shroud along the axial direction. At different positions and different moments, the regularity of velocity distributions between hub and shroud are similar; namely, the velocity is maximum on the wall of hub and shroud, while the velocity is minimum and uniform 


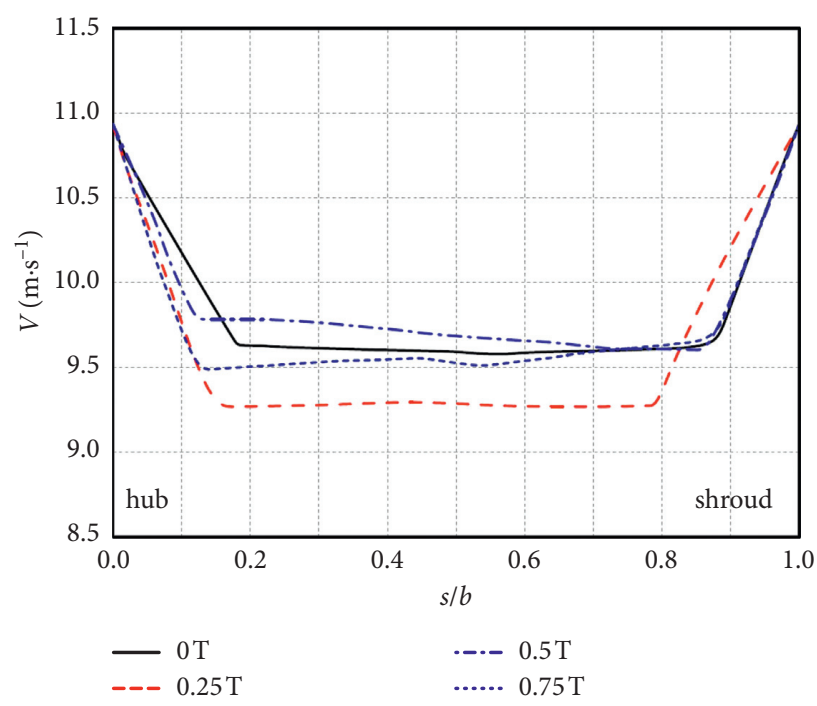

(a)

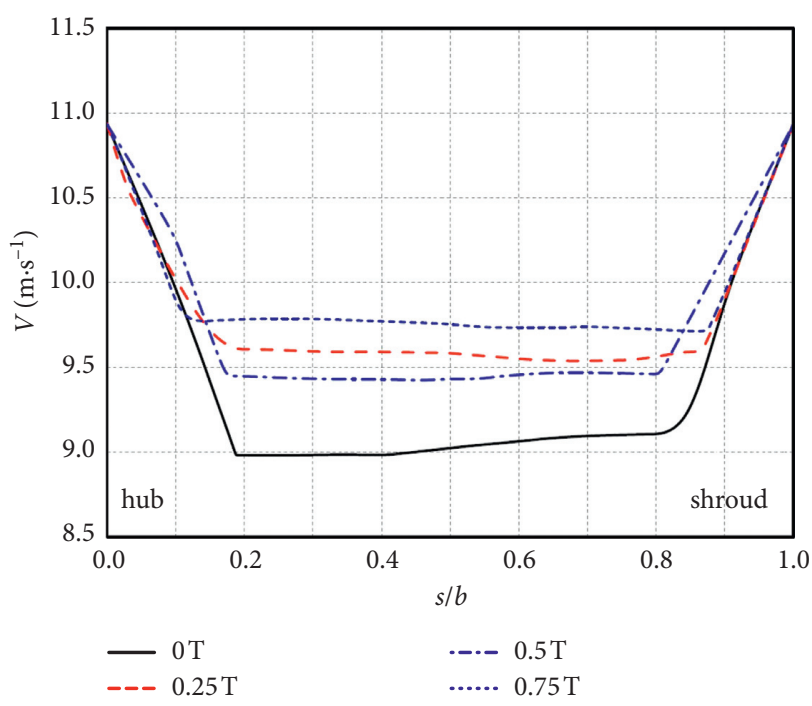

(b)

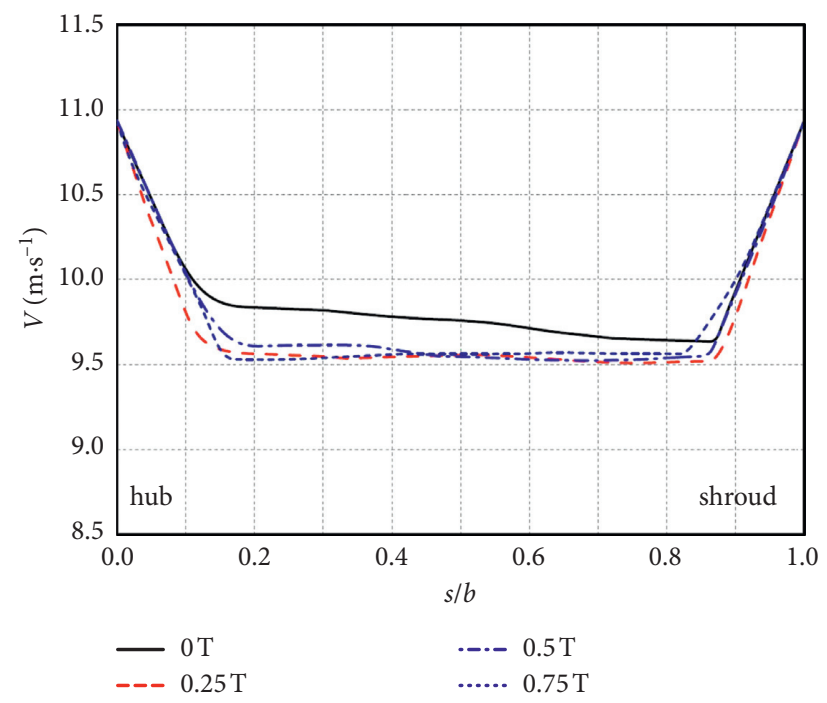

(c)

Figure 3: Velocity distribution between hub and shroud $(r=72 \mathrm{~mm})$. (a) The second section. (b) The fourth section. (c) The sixth section.

distribution at middle positions. The region of stable velocity mainly concentrates on the region of $(0.2 \sim 0.8) b$. Viscosity makes the liquid particle adhere to the wall, so the velocity of the liquid particle is equal to the rotational velocity of the wall. In the vicinity of hub and shroud, there are two linear velocity gradients. Besides the above characteristics, the difference of velocity distributions also exists at different positions and different moments. It is seen from Figure 3 that the average velocities are about 9.59, 9.27, 9.70, and $9.54 \mathrm{~m} / \mathrm{s}$ at $0,0.25,0.50$, and $0.75 \mathrm{~T}$ at the second section. Meanwhile, the average velocities at the fourth section are about 9.03, 9.57, 9.44, and $9.74 \mathrm{~m} / \mathrm{s}$ at $0,0.25,0.50$, and $0.75 \mathrm{~T}$. At four moments and at the sixth section, the average velocities are about $9.75,9.55,9.57$, and $9.56 \mathrm{~m} / \mathrm{s}$.

The velocity distribution of volute inlet-axial at different positions and different moments are shown in Figure 4, the distribution tendency is reverse comparing with the velocity in the impeller. Viscosity makes the liquid particle adhere to the wall; therefore, the velocity of the liquid particle is minimum. The velocity at the middle position is uniform and minimum. In the vicinity of the wall, there are also two linear velocity gradients. No remarkable velocity difference appears at different positions and different moments. It is seen from Figure 4 that the average velocities are about 7.07, $7.42,6.78$, and $6.82 \mathrm{~m} / \mathrm{s}$ at $0,0.25,0.50$, and $0.75 \mathrm{~T}$ at the second section. Meanwhile, the average velocities at the fourth section are about $7.31,7.55,7.67$, and $7.16 \mathrm{~m} / \mathrm{s}$ at 0 , $0.25,0.50$, and $0.75 \mathrm{~T}$. At four moments and the sixth section, the average velocities are about 7.47, 7.56, 7.78, and $7.90 \mathrm{~m} / \mathrm{s}$.

4.2. Closing Valve Characteristics. The comparison between transient process and quasi-steady is shown in Figure 5. It should be noted that the start time of $0.0 \mathrm{~s}$ under this condition is the moment that the working flow rate begins to 


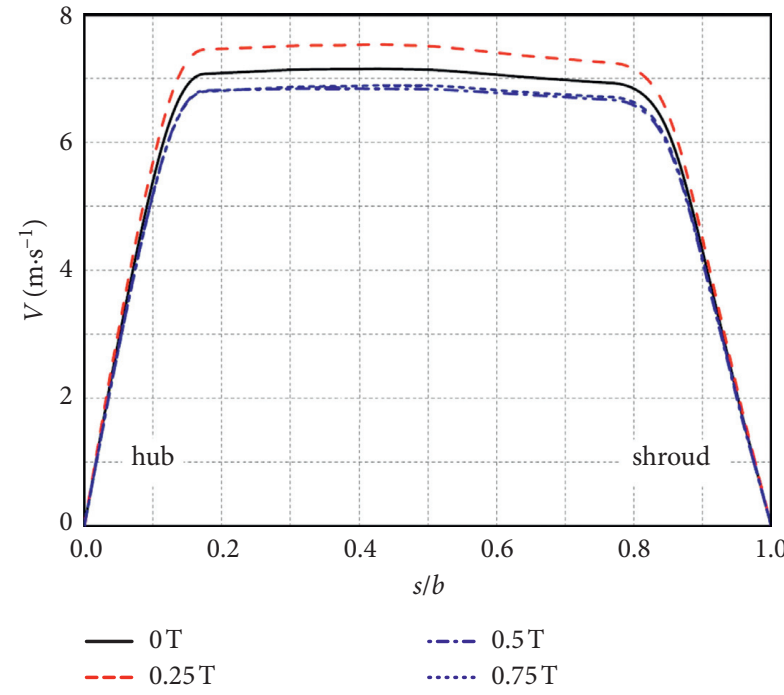

(a)

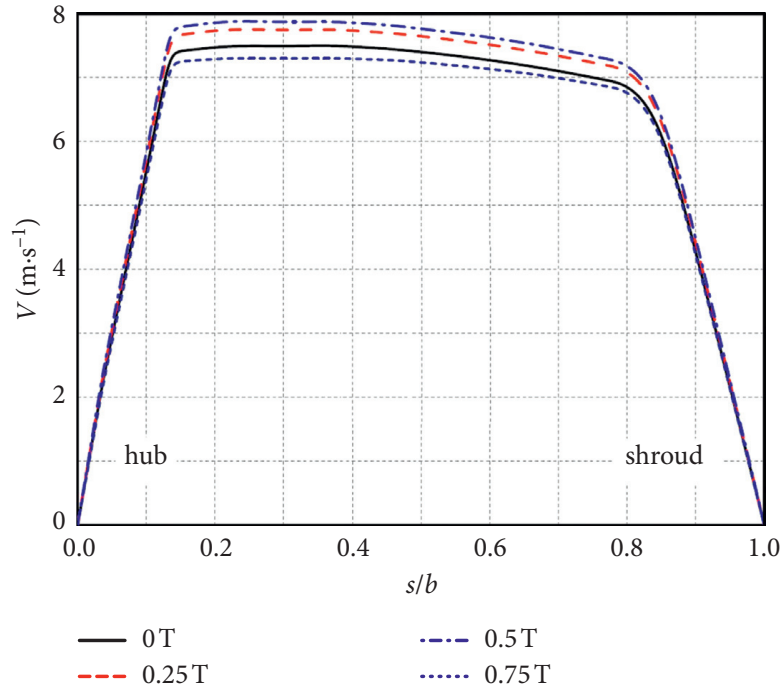

(b)

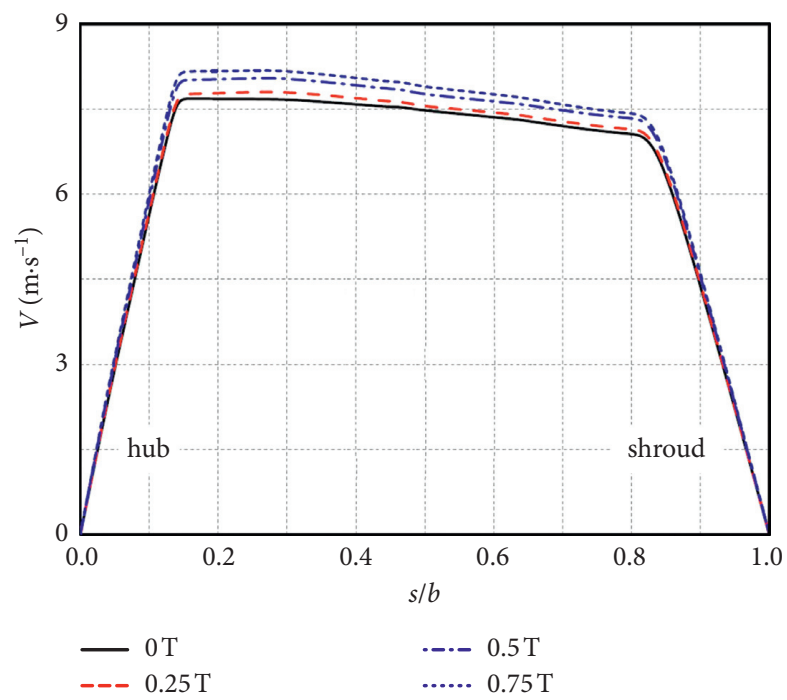

(c)

FIGURE 4: Velocity distribution of volute inlet-axial $(r=82 \mathrm{~mm})$. (a) The second section. (b) The fourth section. (c) The sixth section.

be decreased. As we know, the tongue structure plays an important role in the internal flow field structure. In this paper, the position of tongue structure could be clearly seen in [24]. In quasi-steady calculation, the rotational speed is unchanged and is still $1450 \mathrm{r} / \mathrm{min}$. According to the userdefined function, the corresponding flow rate would be employed to calculate in the quasi-steady. The transient flow field lags behind the quasi-steady flow field, which may be related to flow inertia; namely, kinetic energy cannot convert pressure energy in time. In the process of transient operation, the backflow region first appears in the middle position of the pressure side. Then, with the gradual decrease of flow rate, relative velocity will decrease, the control force of vanes for fluid will be weakened, and flow separation will be more obvious. The backflow region gradually increases, and the eddy center gradually moves towards the impeller outlet. At the impeller inlet, some backflow regions also appear. This backflow will cause plentiful hydraulic loss. The tongue structure makes no backflows in other channels appear. In the process of quasi-steady calculation, two backflow regions appear in the same channel, and with the decrease of flow rate, two backflow regions gradually develop, till full total channel. The velocity vector in a period is shown in Figure 6. The results show that, with the gradual decrease of flow rate, the magnitude of relative velocity also gradually decreases. The pressure at the pressure side is obviously higher than that at the suction side. As such, the relative velocity in the impeller channel would be changed and becomes nonuniform. What is more, the rotor-stator interaction between rotational impeller and stationary volute also plays an important role in jet-wake flow structures. The jet-wake flow structures could be clearly seen in Figure 6. The relative velocity at the pressure side is obviously higher than that at the suction side. The jet-wake flow structures appear at the impeller outlet closing with the volute tongue. In other channels, the relative velocity is uniform, and the jet-wake 


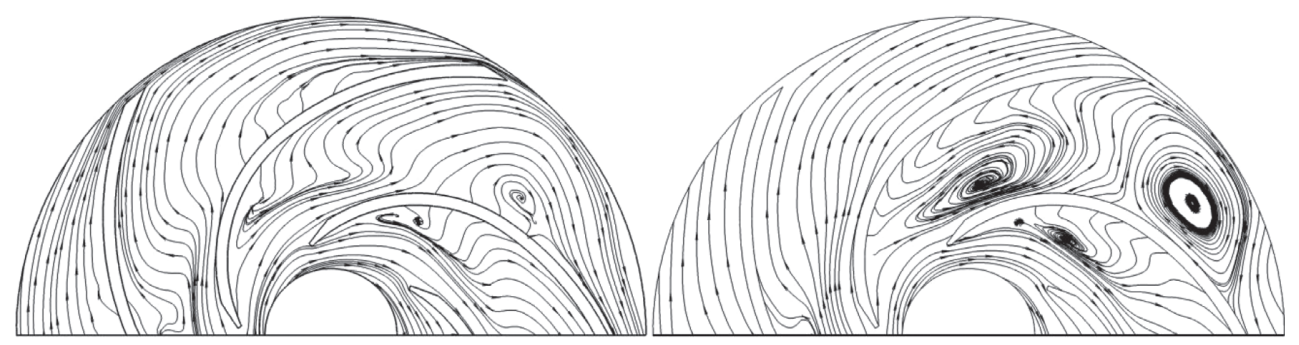

(a)
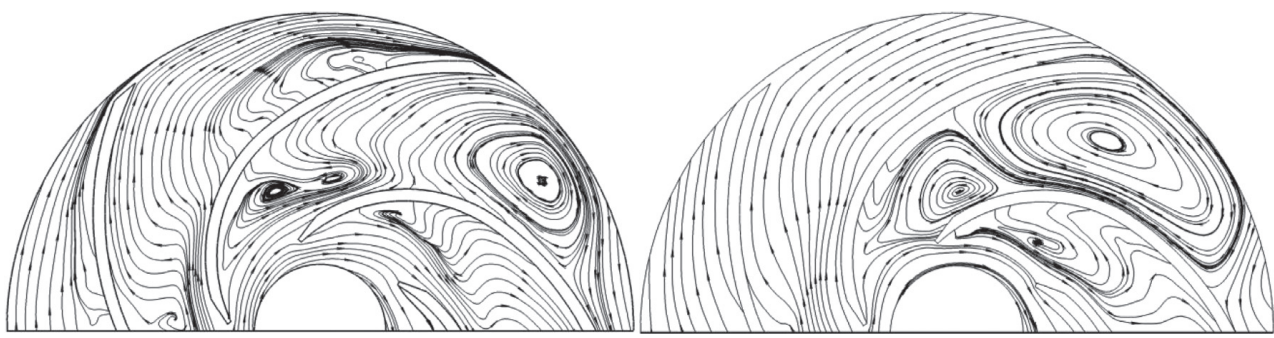

(b)

Figure 5: The comparison of flow field evolution (left: transient; right: quasisteady). (a) $0.027 \mathrm{~s}$; (b) $0.043 \mathrm{~s}$.

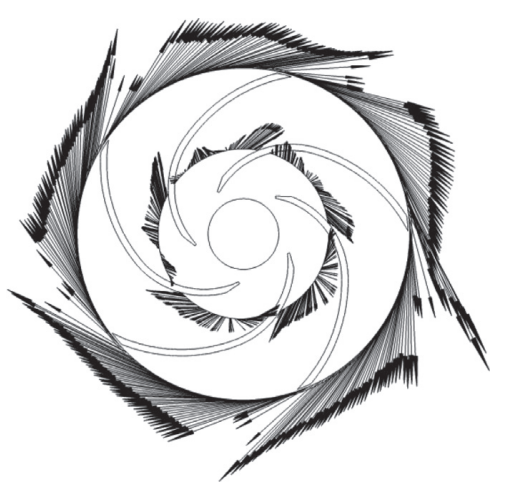

(a)

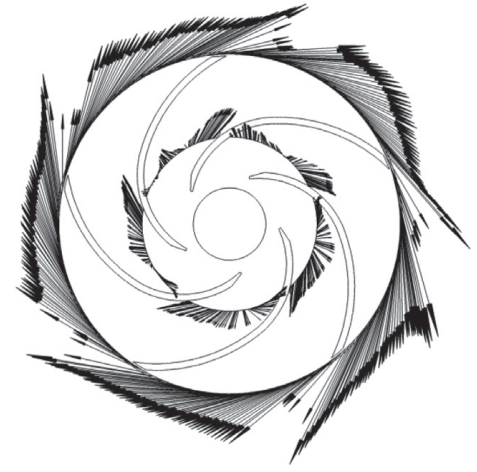

(b)

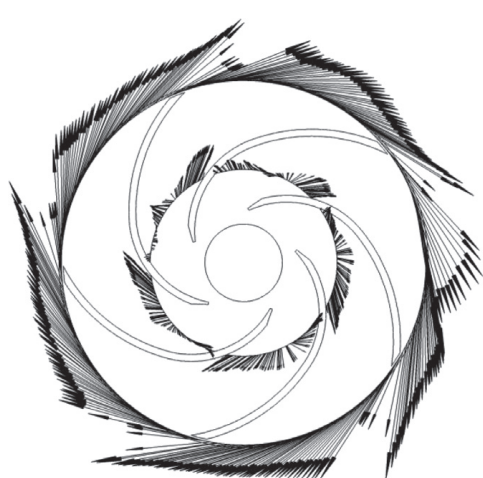

(c)

FIgURE 6: Evolution of velocity vector in a period. (a) 0.029 s. (b) 0.031 s. (c) $0.033 \mathrm{~s}$.

flow structure is not very remarkable. With the change of relative positions between vanes and volute tongue, the jetwake flow structure also will be changed. In the super-lowspecific-speed centrifugal pump, splitter blades often are adopted to weaken the jet-wake flow structure. The calculation results show that besides splitter blades, the geometry parameters and the location parameters of the volute tongue also could be designed to improve the jet-wake flow structure. Relative to the volute tongue, reverse velocity vector appears at the middle of the suction side in the farthest channel; this verifies that backflow region will appear, which may be related to the oversize setting angle at inlet and volute tongue effect.

According to the difference of relative positions between vanes and volute tongue, there are the farthest relative position and the nearest relative position. The pressure distribution at the impeller outlet is shown in Figure 7. The result shows that the distribution regularity is similar. There are obvious periodic characteristics, and the frequency of period characteristics is equal to the blade number. With the gradual decrease of flowrate, the pressures at impeller outlet gradually increase. This characteristic is consistent with the change tendency of external performance of centrifugal pump under target condition. At vanes locations of impeller outlet, there is minimum pressure.

The distributions of pressure coefficient are shown in Figure 8 when the closing valve time is, respectively, 0 Ts and $0.1 \mathrm{~T}$. Definition of pressure coefficient is as follows.

$$
\psi=\frac{p}{2 \rho u_{2}^{2}},
$$

where $u_{2}$ is circular velocity at impeller outlet. Pressure coefficients gradually increase with the augment of radius. At the same radius, the pressure coefficient on pressure sides is obviously higher than that of suction sides. Action force and centrifugal force formed by the pressure difference between the working surface and back surface are consistent with the rotation direction of the centrifugal pump, which completely accords with the reality of the centrifugal pump. There are obvious low-pressure regions at the inlet of suction sides. 


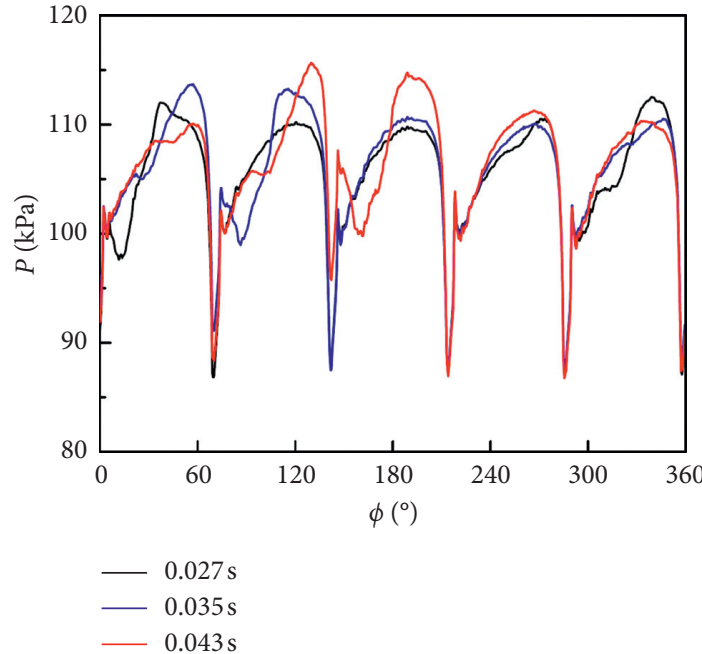

(a)

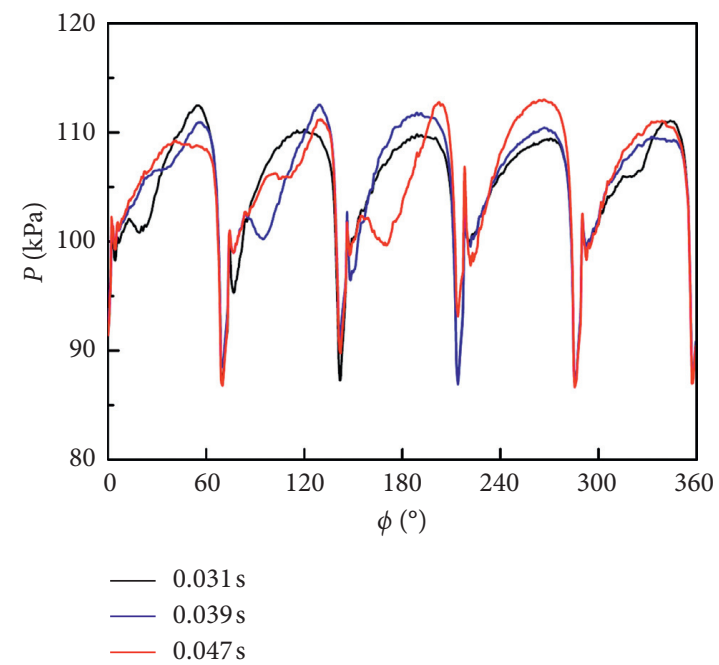

(b)

Figure 7: Pressure distribution at impeller outlet. (a) The relative position is maximum. (b) The relative position is minimum.
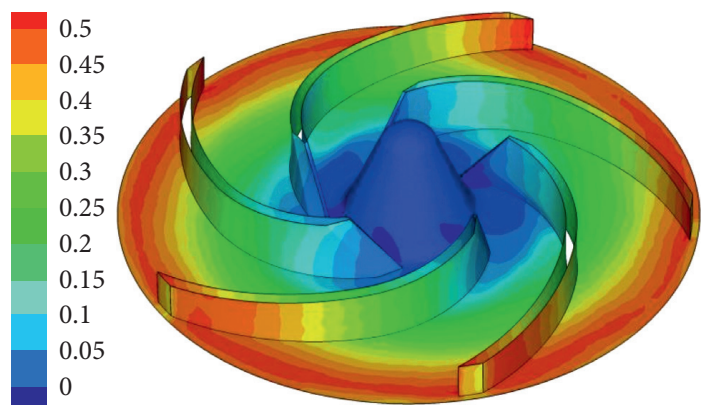

(a)

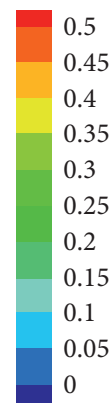

0

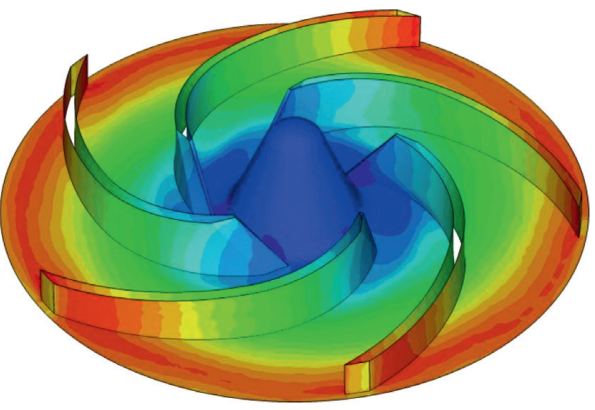

(b)

FIgURE 8: The distribution of pressure coefficient (no shroud). (a) $0.027 \mathrm{~s}$. (b) $0.031 \mathrm{~s}$.

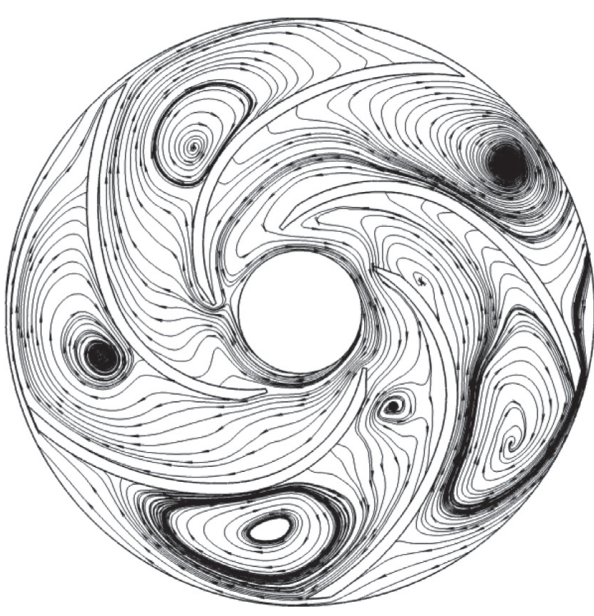

(a)

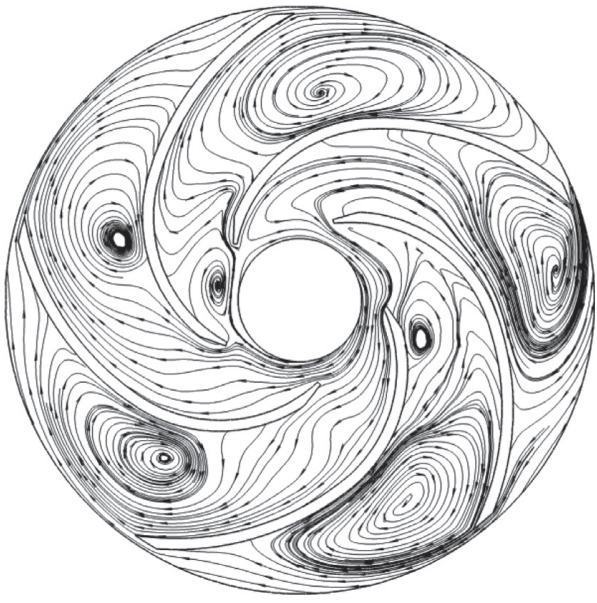

(b)

Figure 9: The streamline distribution under the shutdown state. (a) 0 . (b) $1 / 2 \mathrm{~T}$.

Under cavitation conditions, the cavitation phenomenon will firstly appear at this location. For low rotational speed and high pressure at impeller inlet, the centrifugal pump will not be cavitated, so it is under a good operation state.
4.3. The Characteristics under Shutdown State. The streamline distribution in the centrifugal pump is shown in Figure 9 under the shutdown state. At this time, no flow rate is transported, the rotation of impeller turns into agitation of 
the mixer, so the centrifugal pump becomes a mixer. Agitation action and viscosity action together make plentiful backflow region appear near the suction side in a centrifugal pump.

\section{Conclusions}

The transient behavior of the centrifugal pump is numerically simulated during regulating flowrate valve-off. The velocity distribution regularities are completely reverse in impeller and volute. At the same position, there are slight differences in velocity distribution for different moments in the impeller. The transient flow field lags behind the quasisteady flow field, which may be related to the reasons; namely, kinetic energy cannot convert pressure energy in time. Under shutdown state, centrifugal pump actually becomes a mixer.

\section{Nomenclature}

Q: Volume flow rate

$H$ : Pump head

$n$ : Rotational speed

$s:$ Distance from hub

$b$ : Blade number

$t$ : Time

$p$ : Total pressure at blade surface

$\rho$ : Density of the fluid

$u$ : Circumferential velocity.

Subscripts

0 : Before regulating flow rate

2: Impeller outlet.

\section{Data Availability}

The data used to support the findings of this study are available from the corresponding author upon request.

\section{Conflicts of Interest}

The authors declare no potential conflicts of interest with respect to the research, authorship, and/or publication of this article.

\section{Authors' Contributions}

Yan-Juan Zhao carried out the numerical simulation and wrote the manuscript; Yu-Liang Zhang analyzed the flow characteristics. All the authors have read and agreed to the published version of the manuscript.

\section{Acknowledgments}

The research was financially supported by the National Natural Science Foundation of China (Grants no. 51876103 and 51976202) and Zhejiang Provincial Natural Science Foundation of China (Grant no. LZY21E060001).

\section{References}

[1] H. Tsukamoto and H. Ohashi, "Transient characteristics of a centrifugal pump during starting period," Journal of Fluids Engineering, vol. 104, no. 1, pp. 6-13, 1982.

[2] H. Tsukamoto, S. Matsunaga, H. Yoneda, and S. Hata, "Transient characteristics of a centrifugal pump during stopping period," Journal of Fluids Engineering, vol. 108, no. 4, pp. 392-399, 1986.

[3] H. Tsukamoto, H. Yoneda, and K. Sagara, "The response of a centrifugal pump to fluctuating rotational speed," Journal of Fluids Engineering, vol. 117, no. 3, pp. 479-484, 1995.

[4] P. J. Lefebvre and W. P. Barker, "Centrifugal pump performance during transient operation," Journal of Fluids Engineering, vol. 117, no. 1, pp. 123-128, 1995.

[5] P. Thanapandi and R. Prasad, "Centrifugal pump transient characteristics and analysis using the method of characteristics," International Journal of Mechanical Sciences, vol. 37, no. 1, pp. 77-89, 1995.

[6] A. Dazin, G. Caignaert, and G. Bois, "Transient behavior of turbomachineries: applications to radial flow pump startups," Journal of Fluids Engineering, vol. 129, no. 11, pp. 1436-1444, 2007.

[7] T. Tanaka and H. Tsukamoto, "Transient behavior of a cavitating centrifugal pump at rapid change in operating conditions-Part 1: transient phenomena at opening/closure of discharge valve," Journal of Fluids Engineering, vol. 121, no. 4, pp. 841-849, 1999.

[8] T. Tanaka and H. Tsukamoto, "Transient behavior of a cavitating centrifugal pump at rapid change in operating conditions-Part 2: transient phenomena at pump startup/ shutdown," Journal of Fluids Engineering, vol. 121, no. 4, pp. 850-856, 1999.

[9] T. Tanaka and H. Tsukamoto, "Transient behavior of a cavitating centrifugal pump at rapid change in operating conditions-Part 3: classifications of transient phenomena," Journal of Fluids Engineering, vol. 121, no. 4, pp. 857-865, 1999.

[10] Z. Li, D. Wu, L. Wang, and B. Huang, "Numerical simulation of the transient flow in a centrifugal pump during starting period," Journal of Fluids Engineering, vol. 132, no. 8, pp. 1-8, Article ID 081102, 2010.

[11] D. Wu, L. Wang, and Z. Hu, "Experimental study on explicit performance of centrifugal pump during rapid starting period," Journal of Engineering Thermophysic, vol. 27, pp. 68-70, 2006.

[12] Z. $\mathrm{Hu}, \mathrm{D} . \mathrm{Wu}$, and L. Wang, "Transient hydrodynamic performance of centrifugal pump during rapid starting period: study of explicit characteristics," The Journal of Zhejiang University Science A, vol. 39, pp. 605-608, 2005.

[13] L. Wang, Z. Li, W. Dai, and D. Wu, "2-D numerical simulation on transient flow in centrifugal pump during starting period," Journal of Engineering Thermophysic, vol. 29, pp. 1319-1322, 2008.

[14] D. Wu, B. Xu, Z. Li, and L. Wang, "Numerical simulation on internal flow of centrifugal pump during transient operation," Journal of Engineering Thermophysics, vol. 30, pp. 781-783, 2009.

[15] S. Chen, C. Li, Y. Qu, and R. Sui, "Transient hydraulic performance of a centrifugal pump during rapid starting period," Journal of Engineering Thermophysics, vol. 27, pp. 781-783, 2006.

[16] P. Wu, D. Wu, Z. Li, and L. Wang, "Study of transient flow in centrifugal pump during flow impulsively increase process," 
Journal of Engineering Thermophysic, vol. 31, pp. 419-422, 2010.

[17] M. Liu, L. Tan, and S. Cao, "Influence of geometry of inlet guide vanes on pressure fluctuations of a centrifugal pump," Journal of Fluids Engineering, vol. 140, no. 9, Article ID 091204, 2018.

[18] M. Liu, L. Tan, Y. Xu, and S. Cao, "Optimization design method of multi-stage multiphase pump based on oseen vortex," Journal of Petroleum Science and Engineering, vol. 184, Article ID 106532, 2020.

[19] Y. Han and L. Tan, "Dynamic mode decomposition and reconstruction of tip leakage vortex in a mixed flow pump as turbine at pump mode," Renewable Energy, vol. 155, pp. 725-734, 2020.

[20] W. Xiao and L. Tan, "Design method of controllable velocity moment and optimization of pressure fluctuation suppression for a multiphase pump," Ocean Engineering, vol. 220, Article ID 108402, 2021.

[21] X. Li, T. Shen, P. Li, X. Guo, and Z. Zhu, "Extended compressible thermal cavitation model for the numerical simulation of cryogenic cavitating flow," International Journal of Hydrogen Energy, vol. 45, no. 16, pp. 10104-10118, 2020.

[22] S. Zhang, X. Li, and Z. Zhu, "Numerical simulation of cryogenic cavitating flow by an extended transport-based cavitation model with thermal effects," Cryogenics, vol. 92, pp. $98-104,2018$

[23] Y. Liu, X. Li, M. Ge, L. Li, and Z. Zhu, "Numerical investigation of transient liquid nitrogen cavitating flows with special emphasis on force evolution and entropy features," Cryogenics, vol. 113, Article ID 103225, 2021.

[24] Y. Zhang, J. Xiao, B. Cui, and Z. Zhu, "Simulation of transient behavior in prototype centrifugal pump," Transactions of the CSAE, vol. 30, pp. 68-75, 2014.

[25] I. Gonda, "Experiments on turbulent flow due to an enclosed rotating disk," Experimental Thermal and Fluid Science, vol. 5, pp. 359-368, 1992.

[26] Y. Zhang, Y. Li, B. Cui, Z. Zhu, and H. Dou, "Numerical simulation and analysis of solid-liquid two-phase flow in centrifugal pump," Chinese Journal of Mechanical Engineering, vol. 26, no. 1, pp. 53-60, 2013. 